\title{
Upper Blepharoplasty Scar and Patient Satisfaction Evaluation in a Plastic Surgery Center in Mexico
}

\author{
Victor Hugo Avalos Gómez, Jaime Aron García Espinoza, José Carlos Martínez López, \\ Carlos Illich Navarro Delgadillo, Blanca Yadira Arámbula Sánchez, Alejandro Costa Dulche, \\ Cuahutemoc Márquez Espriella, Esteban Israel Campos Serna
}

Division of Plastic and Reconstructive Surgery, Hospital Central Sur de Alta Especialidad de Petróleos Mexicanos, Mexico City, Mexico

Email: victor_avalosg@hotmail.com

How to cite this paper: Avalos Gómez, V.H., Espinoza, J.A.G., López, J.C.M., Delgadillo, C.I.N., Sánchez, B.Y.A., Dulche, A.C., Espriella, C.M. and Serna, E.I.C. (2020) Upper Blepharoplasty Scar and Patient Satisfaction Evaluation in a Plastic Surgery Center in Mexico. Journal of Biosciences and Medicines, 8, 77-88.

https://doi.org/10.4236/jbm.2020.86008

Received: May 3, 2020

Accepted: June 1, 2020

Published: June 4, 2020

Copyright $\odot 2020$ by author(s) and Scientific Research Publishing Inc. This work is licensed under the Creative Commons Attribution International License (CC BY 4.0).

http://creativecommons.org/licenses/by/4.0/

\begin{abstract}
Background: Eyes are one of the most important facial features, their appearance being associated with state of mind, age and beauty. Therefore, upper blepharoplasty is one of the most common procedures performed in plastic surgery. Standardized tools allow for measurement of outcomes through patient satisfaction and surgeon experience. Objectives: Our objective was to measure the satisfaction index and scar quality of patients who underwent upper blepharoplasty using the Patient-reported Outcome Measure Questionnaire (PROM-Q) and Patient and Observer Scar Assessment Scale (POSAS). Methods: A retrospective review of patients that underwent upper blepharoplasty in our institution was performed. We included those with a preoperative anthropometric analysis, standardized incision markings (developed in our center), same operative technique and a 3-month postoperative evaluation with PROM-Q and POSAS. The sample for this study consists of $67 \mathrm{pa-}$ tients, all of them operated between January 12019 and June 302019 at the Department of Plastic Surgery, South Central High Specialty Hospital, Pemex, Mexico City, Mexico. Results: A total of 67 patients were included, 73\% female and $27 \%$ male. The most frequent indication for surgery was functional visual symptoms. The index of PROM-Q at 3 months demonstrated an increased satisfaction above $90 \%$, and the evaluation with POSAS reported an almost imperceptible scar. Conclusion: Evaluating outcomes with objective tools provides data useful to improve the surgical protocols of patients subjecting to aesthetic procedures.
\end{abstract}

\section{Keywords}

Upper Blepharoplasty, Satisfaction Index, PROM-Q, Quality of Life, Scar 


\section{Introduction}

Upper blepharoplasty is one of the most common procedures performed in plastic surgery around the world. The International Society of Aesthetic Plastic Surgery (ISAPS) reported it to be the third most frequent procedure, with an increasing demand of $6 \%$ every year. $85 \%$ of patients subjected to it are women and $15 \%$ men, ranging from 50 to 70 years old [1]. The goal of the procedure is the restoration of a youthful appearance, accentuation of the supratarsal crease, correction of contour deformities, fat pad herniation and rhytids [2]. Aside from the cosmetic value, its functional benefits are the improvement of the superior field of vision and ptosis in some cases, by decreasing the weight borne by the levator palpebrae superioris [3].

Current research is focusing on evaluating short- and long-term outcomes, along with obtaining objective measurements of postoperative satisfaction and quality of life. In 1990, Putterman [4] reported that satisfaction was directly correlated with adequate doctor patient relationship, and more recently, Gladstone [5] added that it directly depended on knowing patient expectations and a good perioperative experience. In the past few years, multiple authors have encouraged surgeons to adopt a stepwise approach for preoperatory evaluation. This includes asking the patient for pictures of their youth, performing a proper anthropometric analysis of the eyelids, purposefully searching for hidden ptosis and evaluating eyebrow position [6].

Most authors agree that precise geometrical incision markings that consider the patient's race and individual characteristics improve outcomes. Some of the key points are preserving at least $10 \mathrm{~mm}$ of pretarsal skin and $10 \mathrm{~mm}$ of skin below the brow line, avoiding extending an incision medial to the punctum or 10 $\mathrm{mm}$ lateral to the external canthus [7]. A matter still in question is the extent of the impact of preseptal orbicularis oculi muscle resection in patient satisfaction. Despite the efforts, further study is needed [8]. Recently, more studies are concentrating in improving perioperative patient experience and preventing outcomes that predispose to dissatisfaction (partial or complete loss of pretarsal crease, hypertrophic scars, asymmetry, lagophthalmos, ptosis and hematomas) [9].

Despite the efforts to introduce scales that measure the index of satisfaction, there are still deficiencies in their implementation and objective analysis. Recently, the Royal College of Surgeons of England developed the PROM-Q [10], a validated scale that determines the satisfaction index in patients that have undergone blepharoplasty, which takes into consideration the subjective experience of adverse symptoms (dry eyes, eye irritation, excessive tearing, etc.) and the final appearance of the eyelids after surgery.

Another promising tool that evaluates scars, both objectively and subjectively, is the Patient and Observer Scar Assessment Scale (POSAS). In 2003, Lieneke [11] compared it with the Vancouver scale, and found it to have a lesser interobserver variability ( $18 \%$ vs. $22 \%)$ and a higher interclass correlation coefficient (0.92 vs. 0.90), proving it to be a promising instrument that allows for the evalu- 
ation of scars in a more objective manner with less variance. Despite these results, no studies have been published about using this scale for the evaluation of upper blepharoplasty scar.

\section{Objectives}

To measure the satisfaction index and scar quality of patients who underwent upper blepharoplasty using the PROM-Q and POSAS, as well as the measurement of analgesic control using Visual Analogue Scale (VAS), and the epidemiological characteristics of our population.

\section{Materials and Methods}

A review of medical records of patients that underwent upper blepharoplasty in our plastic surgery center was performed. The study included patients with a complete photographic record (frontal preoperative and postoperative photographs focused on the upper third of the face, standardized 1 meter away with patient standing upright in neutral gaze), and preoperative anthropometric analysis based on a checklist developed in our center (static and dynamic measurement of the upper eyelid and brow) (Appendix 1). All surgeries were performed in an outpatient setting under local anesthesia with sedation. Surgical technique included cutaneous incision following standardized incision markings designed by our plastic surgery team (Image 1), resection of skin and a strip of pre-septal orbicularis oculi muscle, resection of herniated upper central and nasal fat pad, strict hemostasis and wound closure with subcuticular running 6-0 polypropylene suture. During follow up, postoperative pain was measured with VAS on days 3, 7 and 15. Stiches were removed on day 5, and after 3 months, PROM-Q (Appendix 2) and POSAS (Appendix 3) were filled and final postoperative photographs were taken, all by the same two investigators. The sample for this study consists of 67 patients, all of them operated between January 1 2019 and June 302019 at the Department of Plastic Surgery, South Central High Specialty Hospital, Pemex, Mexico City, Mexico.

Patient satisfaction is measured using the postoperative PROM-Q questionnaire. The questionnaire is divided in two sections, satisfaction with eyes, and symptoms checklist. For the satisfaction with eyes section, each answer is rated with the following points scale: 1 point for very dissatisfied, 2 points for somewhat dissatisfied, 3 points for somewhat satisfied and 4 points for very satisfied. In this section, when obtaining a total score from 7 to 14 points, the results are deemed unsatisfactory and from 15 to 28 points, results are deemed satisfactory.

Regarding the symptoms checklist section, the rating is as follows: 1 point when the answer is Not at all, 2 points for A little, 3 for Moderately, and 4 for Extremely. In this section, when obtaining a total score from 6 to 17 the results are deemed as few symptoms results. When obtaining a score of 18 to 24 , the results are considered as very intense symptoms.

In the POSAS scale, the Observer section is dived in 6 items, each item with a 


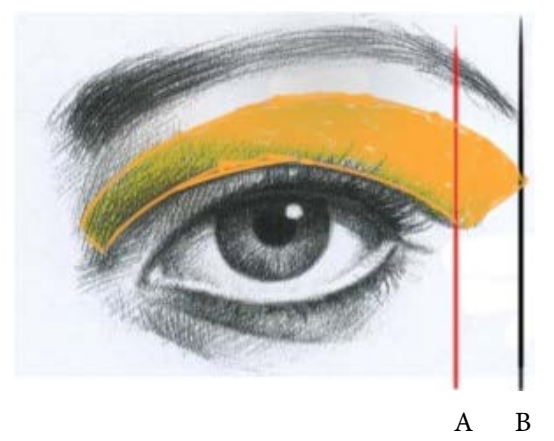

Image 1. Gurtierrez's upper blepharoplasty surgical markings. Two vertical lines are drawn, one in the lateral orbital margin (A) and a second one at the level of the lateral end of the brow (B). Inferior incision is planned over the native palpebral crease or 10 $\mathrm{mm}$ above the line of the eyelashes, when it reaches line " $\mathrm{A}$ " it follows a $45^{\circ}$ oblique course towards line " $\mathrm{B}$ ". If it is the case, over a rhytid. The upper incision is drawn $10 \mathrm{~mm}$ below the brow line, starting in the medial end of the inferior incision and ending in the convergence of the inferior incision and the B line. After markings are done, always perform a pinch test to avoid lagophthalmos (this technique was developed by one of the teachers of the plastic surgery course in our unit).

rating from 1 to 10 , where 1 is a normal skin and 10 is for worst scar imaginable. Results with total score from 6 to 18 , are considered as a normal skin, results from 19 to 30 are for similar to the skin, from 31 to 42 are for noticeable differences, 43 to 54 are for different and, 55 to 60 for very different skin.

In the same scale, the patient section is divided in 7 items, also rating from 1 to 10 . A total score from 7 to 21 is considered as ideal results with no symptoms, from 22 to 35 good results with mild symptoms, from 36 to 49 non frequent noticeable symptoms, from 50 to 63 mild symptoms all the time, and from 64 to 70 important and frequent symptoms affecting quality of life.

Epidemiological variables included were age, sex, and postoperative medication (type of antibiotics, analgesics and length of treatment).

\section{Results}

A total of 67 patients were included, with an average age of 64.5 years. $73 \%$ were women and $27 \%$ men. Both groups were of similar ages, predominantly towards the $7^{\text {th }}$ decade of life (Table 1). The main indication for surgery was functional, with a slightly higher tendency in the aesthetic reasons in the female (Figure 1). During follow up, $73 \%$ of patients received antibiotics $\left(2^{\text {nd }}\right.$ generation cephalosporins) for 5 days; $100 \%$ of the patients received pain medication (of which the most common prescription was acetaminophen, 87\%) that was continued for 3 days in $95 \%$ of the patients and 5 days in $5 \%$. The pain assessment with VAS showed a drastic decrease between day 3 and $14^{\text {th }}$, when it was qualified as nearly absent. The female group perceived a higher degree of pain than males (Figure 2).

The satisfaction index measured with PROM-Q showed an average of 26.06 points (Satisfied range). However, when comparing the male and female groups, the males displayed a lower value of total satisfaction, nevertheless still remaining within the satisfaction range (Figure 3 ). When analyzing questionnaire va- 
riables individually, "bright-eyed look" scored the least points in contrast to "open look" that scored the highest satisfaction (Figure 4).

Table 1. Age by sex.

\begin{tabular}{ccc}
\hline & Average age & ICR \\
\hline Total & 64.5 & $58.5-71$ \\
Male & 65.7 & $65.2-68.2$ \\
Female & 65.8 & $56.5-73$ \\
\hline
\end{tabular}

Surgery Indication (\%)

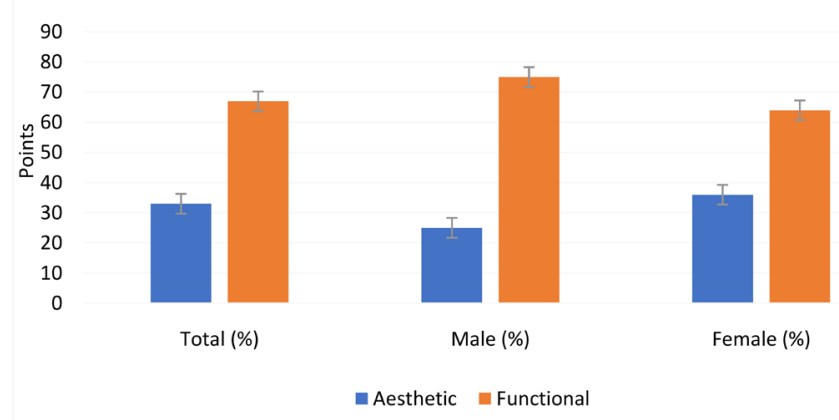

Figure 1. Indication for surgery.

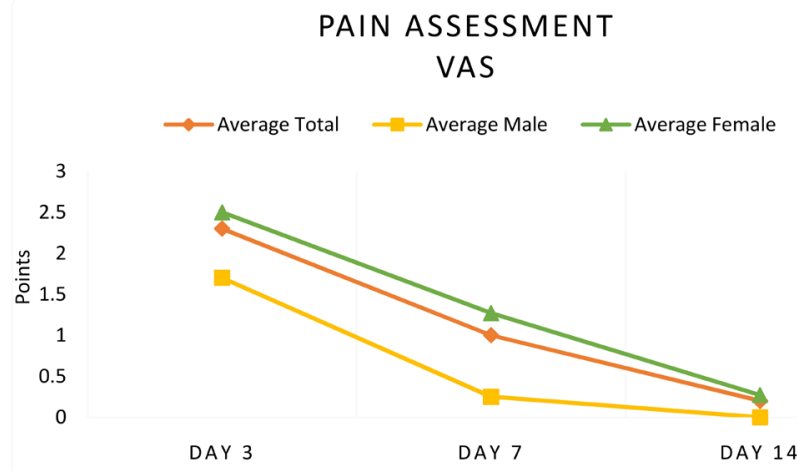

Figure 2. Pain assessment with VAS.

PROM-Q: Satisfaction Index Score

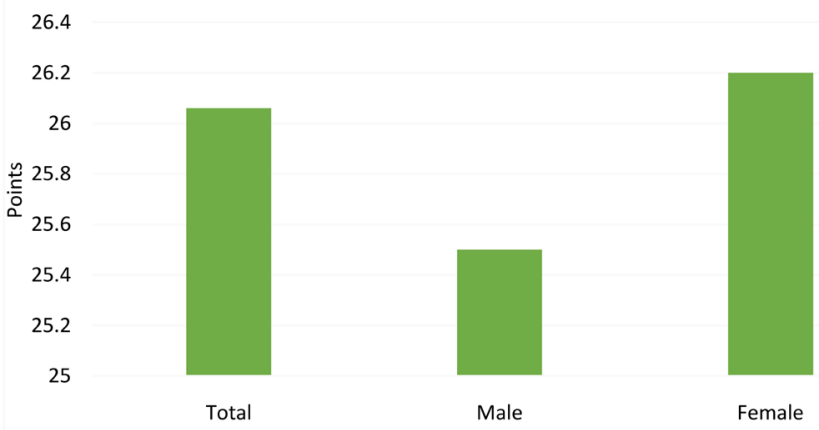

Figure 3. PROM-Q satisfaction index total score. 
PROM-Q: Individual Item Satisfaction

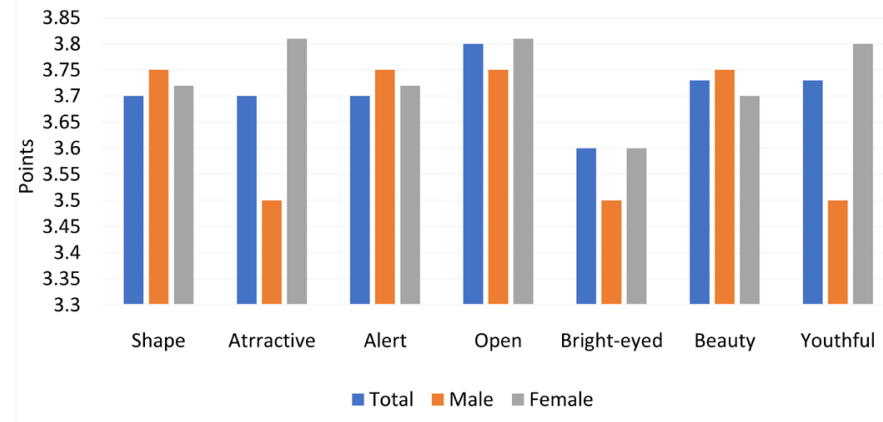

Figure 4. PROM Q-individual item satisfaction.

Patients experienced an average of 9.7 points (minimal to null range) amongst adverse symptoms, with a slightly higher score for the male group (Figure 5). Individually, "Dry eyes" and "Eye irritation" were the most prevalent symptoms with a similar distribution amongst the two groups (Figure 6). The scar evaluation with POSAS, showed an observer average of 11.26 points (almost imperceptible scar), with a slightly lower score in the male group compared to the average of the population (Figure 7). The items that obtained a higher score overall were those related to scar appearance, though all of them ranked a high satisfaction index (Figure 8). No immediate or late complications were reported by any patient.

\section{Discussion}

In plastic surgery it is crucial not only to subjectively assess the benefits of upper blepharoplasty, but to objectively determine improvements and patient perception using validated scales. The PROMs objectively measure the direct impact of the treatment on the patient and allow us to develop health policies for every day practice [12]. These types of questionnaires began in 1950 and caused controversy because they introduced the patient's experience and point of view into health care [13].

In 2001 Bullock et al. [14] found that upper eyelid dermatochalasis had a negative impact in quality of life and perception of self-image in the group of patients studied; furthermore, Kosowsky [15] demonstrated in his study that PROMs are important to establish improvements in perioperative treatment of patients who underwent upper blepharoplasty. One of the most important items evaluated in this questionnaire is anthropometric measurements of the eyelid in order to plan precise markings and optimize results. A study conducted by Sarwer [16] showed a satisfaction rate above $87 \%$ after upper blepharoplasty regardless of the technique performed. In our study we standardized the preoperatory evaluation of anthropometric measurements and markings following $\mathrm{Gu}-$ tierrez's technique. All patients had the stitches removed on post operatory day 5 and a satisfaction evaluation was performed 3 months after surgery. This evalua- 
tion found a high index of satisfaction amongst our patients (according to PROM-Q) that correlates with what is published in the literature by Jacobsen [7] and Jhosi [17]; from a possible 28 points, we obtained an average of 26 points in our series.

POSAS: Complication Symptoms Score

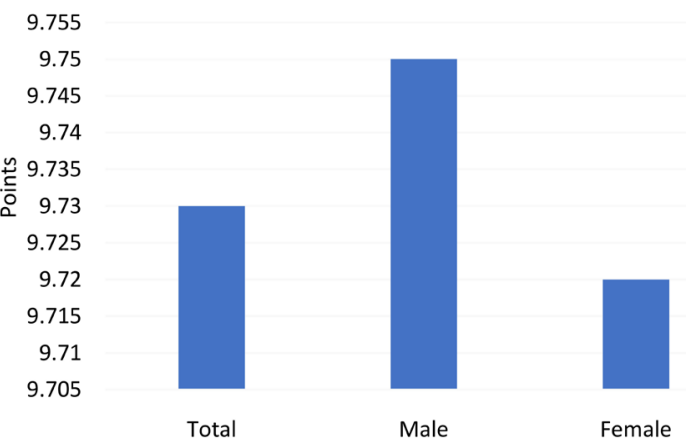

Figure 5. POSAS: complication symptoms score.

PROM-Q: Individual Symptoms Score

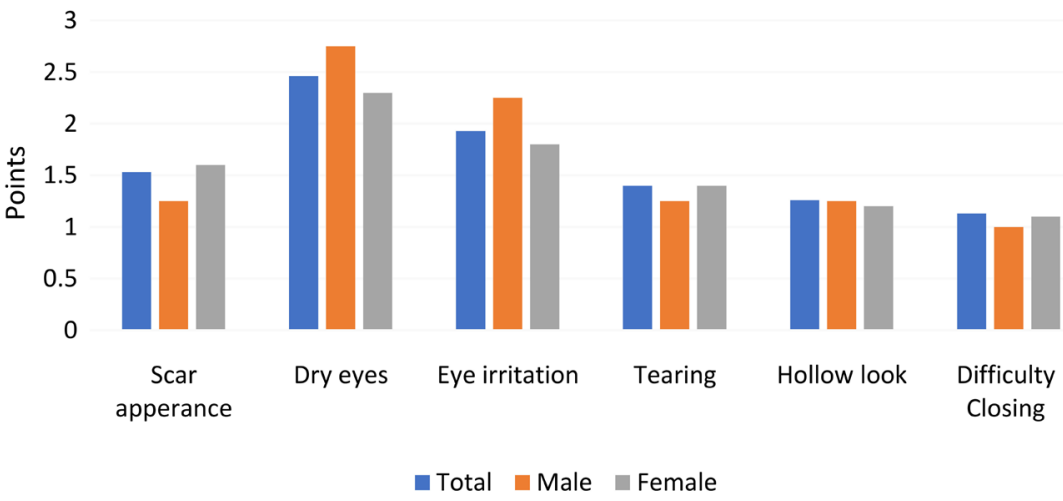

Figure 6. PROM-Q: individual symptoms score.

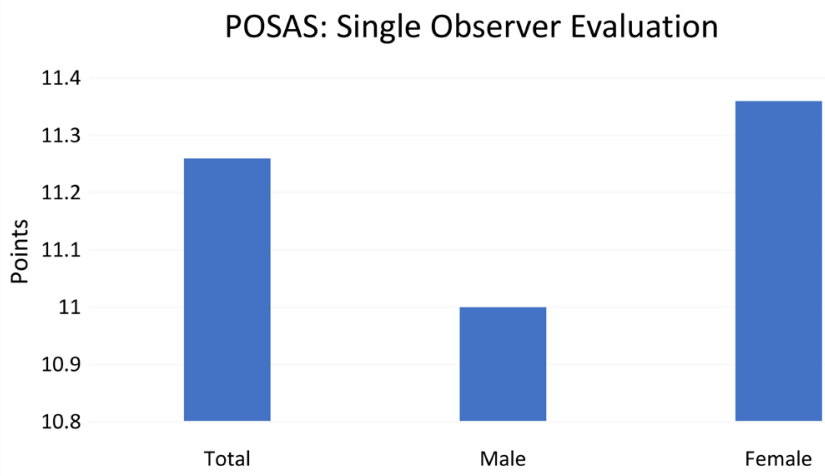

Figure 7. POSAS: single observer evaluation (global and by sex). 


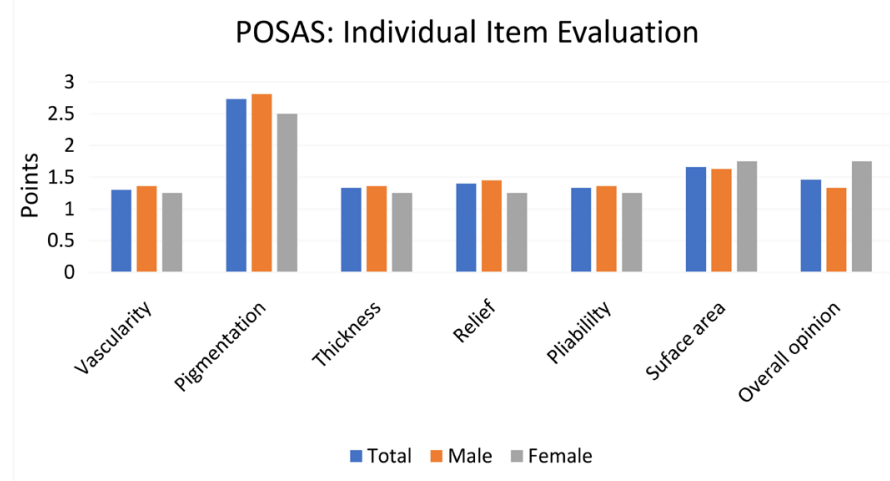

Figure 8. POSAS: individual item evaluation.

When each item of the satisfaction questionnaires was assessed individually, they were found to also have high rates of postoperative satisfaction. Regarding patient experience of adverse symptoms, an average of 9.7 points was recorded, that falls into the low or null range, demonstrating a better experience and high satisfaction with the results. This high level of satisfaction after upper blepharoplasty is also related to the improvement of the look of the eyes (one of the facial characteristics that stands out the most), and correcting dermatochalasis and fat herniation results in a younger, aesthetic and less fatigued look [18]. Therefore, the use of a standardized technique for preoperative analysis, planning and procedure, allows us to achieve higher rates of satisfaction by decreasing errors in diagnosis and postoperative complications.

One of the aspects evaluated in our study was the surgeon's perspective of the final appearance of the scar using the POSAS scale. This tool has been validated and proven to be superior to other scales (Vancouver), as well as being useful to evaluate new ones, yet it is not widely adopted [19]. The single observer evaluation yielded an average score of 11.26 points, which demonstrates a nearly imperceptible scar with very high symmetry between both eyelids. Regarding this, Kouba [20] and Joshi [17] assessed the scars of patients that underwent upper blepharoplasty, nevertheless no standardized tools were used for evaluation. In our study, POSAS allowed for an objective and standardized measurement of every aspect of the scar. Few other studies have routinely implemented this. It is a promising tool for objective postoperative evaluation, yet more studies are necessary to make its use standard in blepharoplasty scars.

\section{Conclusions}

The objective evaluation of outcomes after blepharoplasty is fundamental in everyday practice, as it allows for analysis from surgeon and patient's perception (POSAS and PROM-Q). Performing satisfaction index studies with this type of standardized tools allows us to create systematized attention protocols that improve outcomes and perioperative patient experience. The standardization of preoperative evaluation, surgical technique and postoperative treatment, is intended to reduce complications to the maximum, the single and most important 
factor related to patient dissatisfaction after surgery. Therefore, satisfaction evaluation in plastic surgery is currently a cornerstone factor in the race to improve patient treatment.

We believe it is fundamental to continue to pursue the development of standardized scales such as PROM-Q and POSAS, as they will provide us with information that will better our surgical planning and techniques.

\section{Limitation of the study}

This study was performed over a heterogeneous population following the order they entered to the medical procedures. The study analyses only the perception about symptoms and satisfaction, which can be different between individuals. Hence, the study reflects the perception of this group. The size of the sample is also small, only 67 individuals. A larger sample would be required to conclude and validate the results.

\section{Compliance with Ethical Standards}

1) This work was submitted and approved by the ethics committee of our hospital headquarters with number 51/19.

2) For this type of study informed consent is not required.

\section{Conflicts of Interest}

The authors declare that they have no conflicts of interest to disclose.

\section{References}

[1] American Society of Plastic Surgeons. 2013 Top Five Cosmetic Surgical Procedures. https://www.isaps.org/wp-content/uploads/2019/12/ISAPS-Global-Survey-Results-2 018-new.pdf

[2] Codner, M.A., Kikkawa, D.O., Korn, B.S. and Pacella, S.J. (2010) Blepharoplasty and Brow Lift. Plastic and Reconstructive Surgery, 126, 1e-17e. https://doi.org/10.1097/PRS.0b013e3181dbc4a2

[3] Zoumalan, C.I. and Roostaeian, J. (2016) Simplifying Blepharoplasty. Plastic and Reconstructive Surgery, 137, 196e-213e. https://doi.org/10.1097/PRS.0000000000001906

[4] Putterman, A.M. (1990) Patient Satisfaction in Oculoplastic Surgery. Ophthalmic Surgery, Lasers and Imaging Retina, 21, 15-21.

[5] Gladstone, H.B. (2005) Blepharoplasty: Indications, Outcomes, and Patient Counseling. Skin Therapy Letter, 10, 4-7.

[6] Parbhu, K.C., Hawthorne, K.M., McGwin Jr., G., Vicinanzo, M.G. and Long, J.A. (2011) Patient Experience with Blepharoplasty. Ophthalmic Plastic \& Reconstructive Surgery, 27, 152-154.

[7] Jacobsen, A.G., Brost, B., Vorum, H. and Hargitai, J. (2017) Functional Benefits and Patient Satisfaction with Upper Blepharoplasty-Evaluated by Objective and Subjective Outcome Measures. Acta Ophthalmologica, 95, 820-825. https://doi.org/10.1111/aos.13385

[8] Hoorntje, L.E., Lei, B.V., Stollenwerck, G.A. and Kon, M. (2010) Resecting Orbicu- 
laris Oculi Muscle in Upper Eyelid Blepharoplasty-A Review of the Literature. Journal of Plastic, Reconstructive \& Aesthetic Surgery, 63, 787-792. https://doi.org/10.1016/j.bjps.2009.02.069

[9] Patrocinio, T.G., Patrocinio, L.G. and Patrocinio, J.A. (2018) Effect of Orbicularis Muscle Resection during Blepharoplasty on the Position of the Eyebrow. Facial Plastic Surgery, 34, 178-182. https://doi.org/10.1055/s-0038-1636904

[10] Klassen, A.F., Cano, S.J., Schwitzer, J.A., Scott, A.M. and Pusic, A.L. (2015). FACE-Q Scales for Health-Related Quality of Life, Early Life Impact, Satisfaction with Outcomes, and Decision to Have Treatment: Development and Validation. Plastic and Reconstructive Surgery, 135, 375-386. https://doi.org/10.1097/PRS.0000000000000895

[11] Draaijers, L.J., Tempelman, F.R., Botman, Y.A., Tuinebreijer, W.E., Middelkoop, E., Kreis, R.W., et al. (2004) The Patient and Observer Scar Assessment Scale: A Reliable and Feasible Tool for Scar Evaluation. Plastic and Reconstructive Surgery, 113, 1960-1966. https://doi.org/10.1097/01.PRS.0000122207.28773.56

[12] Nelson, E.C., Eftimovska, E., Lind, C., Hager, A., Wasson, J.H. and Lindblad, S. (2015) Patient Reported Outcome Measures in Practice. BMJ, 350, 1-3. https://doi.org/10.1136/bmj.g7818

[13] Braithwaite, T., Calvert, M., Gray, A., Pesudovs, K. and Denniston, A.K. (2019) The Use of Patient-Reported Outcome Research in Modern Ophthalmology: Impact on Clinical Trials and Routine Clinical Practice. Patient Related Outcome Measures, 10, 9-24. https://doi.org/10.2147/PROM.S162802

[14] Bullock, J.D., Warwar, R.E., Bienenfeld, D. G., Marciniszyn, S.L. and Markert, R.J. (2001) Psychosocial Implications of Blepharoptosis and Derma-Tochalasis. Transactions of the American Ophthalmological Society, 99, 65-71.

[15] Kosowski, T.R., McCarthy, C., Reavey, P.L., et al. (2009) A Systematic Review of Patient-Reported Outcome Measures after Facial Cosmetic Surgery and/or Nonsurgical Facial Rejuvenation. Plastic and Reconstructive Surgery, 123, 1819-1827. https://doi.org/10.1097/PRS.0b013e3181a3f361

[16] Sarwer, D.B., Gibbons, L.M., Magee, L., et al. (2005) A Prospective, Multi-Site Investigation of Patient Satisfaction and Psychosocial Status Following Cosmetic Surgery. Aesthetic Surgery Journal, 25, 263-269. https://doi.org/10.1016/j.asj.2005.03.009

[17] Joshi, A.S., Janjanin, S., Tanna, N., Geist, C. and Lindsey, W.H. (2007) Does Suture Material and Technique Really Matter? Lessons Learned from 800 Consecutive Blepharoplasties. Laryngoscope, 117, 981-984.

https://doi.org/10.1097/MLG.0b013e31804f54bd

[18] Saalabian, A.A., Liebmann, P. and Deutinger, M. (2017) Which Tissue Should Be Removed in Upper Blepharoplasty? Analysis and Evaluation of Satisfaction. World Journal of Plastic Surgery, 6, 324-331.

[19] Bae, S.H. and Bae, Y.C. (2014) Analysis of Frequency of Use of Different Scar Assessment Scales Based on the Scar Condition and Treatment Method. Archives of Plastic Surgery, 41, 111-115. https://doi.org/10.5999/aps.2014.41.2.111

[20] Kouba, D.J., Tierney, E., Mahmoud, B.H. and Woo, D. (2011) Optimizing Closure Materials for Upper Lid Blepharoplasty: A Randomized, Controlled Trial. Dermatologic Surgery, 37, 19-30. https://doi.org/10.1111/j.1524-4725.2010.01834.x 


\section{Appendix 1. Preoperative Anthropometric Analysis Checklist}

Division of Plastic and Reconstructive Surgery

South Central High Specialty Hospital, PEMEX

Brow Position

Distance Brow-Hairline

$(5-6 \mathrm{~cm})$

Distance Brow-Orbital Rim

$(0.5-1 \mathrm{~cm})$

Upper Eyelid Anthropometric Analysis

Static Analysis

Palpebral Fissure (Height)

Palpebral Fissure (Width)

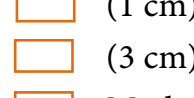

Palpebral Rhytids: Minor

Moderate

Nasal Fat Pad: Normal

Central Fat Pad: Normal

Herniated

Orbicularis Muscle: Normal

Lacrimal Gland: Normal

Tarsal Crease:

\section{Herniated}

Hypertrophy

Dynamic Analysis

MRD 1:

$(4 \mathrm{~mm})$

Upper Eyelid Excursion:

$(10-15 \mathrm{~mm})$

Hering's Test (Contralateral Ptosis):

- Right: Positive
$\square$ Negative
Negative

Left: Positive

\section{Appendix 2. Outcome Measures for Cosmetic Surgery: Postoperative Q-PROM Data Collection Tools}

Q-PROM postoperative questionnaire

FACE-Q-Blepharoplasty

\section{Satisfaction with eyes}

With your eyes in mind, in the past week, how dissatisfied or satisfied have you been with:

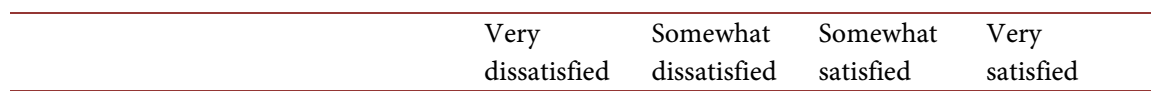

1) The shape of your eyes?

2) How attractive your eyes look?

3) How alert (not tired) your eyes look?

4) How open your eyes look?

5) How bright-eyed you look?

6) How nice your eyes look?

7) How youthful your eyes look?

Scoring key: Very dissatisfied $=1$, somewhat dissatisfied $=2$, somewhat satisfied $=3$, very satisfied $=4$.

\section{Symptom checklist-eyes}

These questions ask about problems you may be experiencing.

With your eyes in mind, in the past week, how much have you been bothered 
by:

\begin{tabular}{lllll}
\hline & Not at all & A little & Moderately & Extremely \\
\hline $\begin{array}{l}\text { 1) How your eyelid scars look } \\
\text { (obvious, noticeable, uneven)? }\end{array}$ & 1 & 2 & 3 & 4 \\
2) Dry eyes? & 1 & 2 & 3 & 4 \\
3) Eye irritation (e.g. redness, itching)? & 1 & 2 & 3 & 4 \\
4) Excessive tearing? & 1 & 2 & 3 & 4 \\
5) Your eyes looking hollowed out? & 1 & 2 & 3 & 4 \\
6) Difficulty closing your eyes? & 1 & 2 & 3 & 4 \\
\hline
\end{tabular}

Appendix 3. The Patient and Observer Scar Assessment Scale V2.0

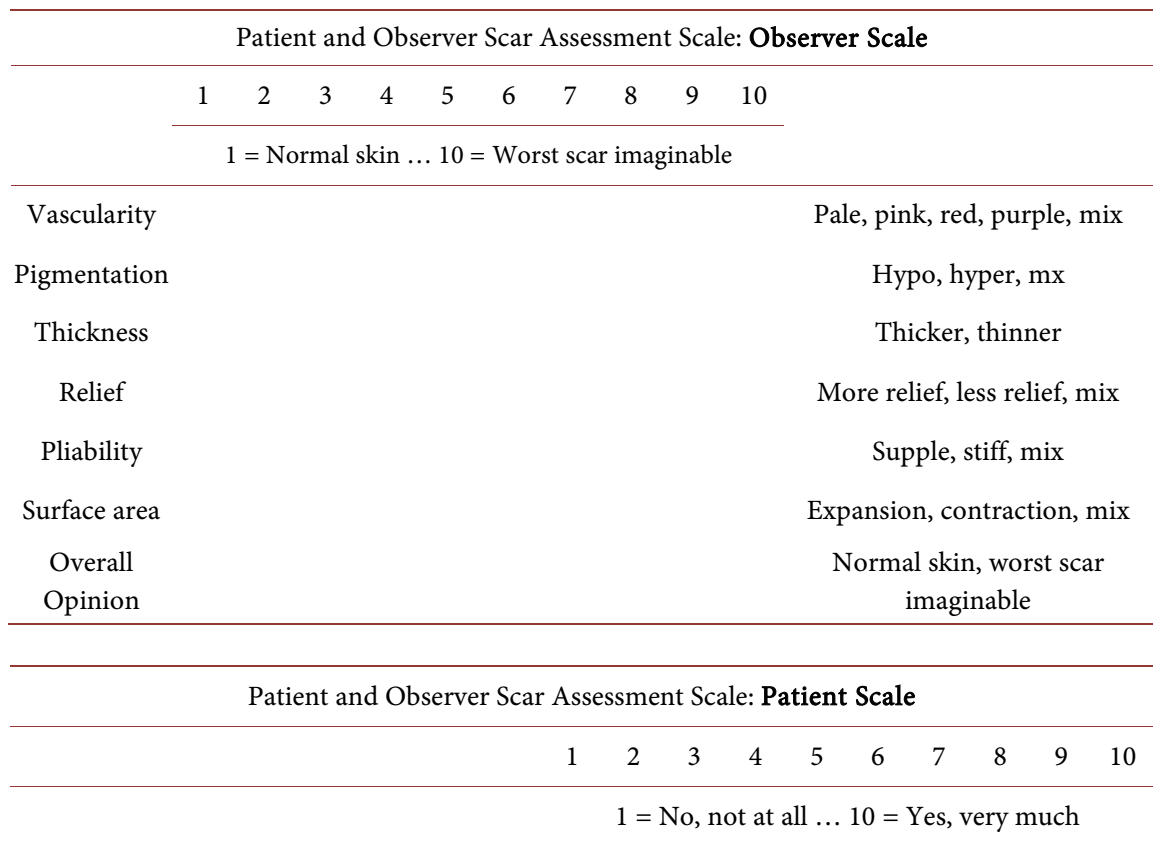

Has the scar been painful the past few weeks?

Has the scar been itching the past few weeks?

$1=$ No, as normal skin $\ldots 10=$ Yes, very different

In the scar color different from the color of

your normal skin at present?

Is the stiffness of the scar different from your

normal skin at present?

Is the thickness of the scar different from your

normal skin at present?

Is the scar more irregular than your normal skin at present?

$1=$ As normal skin $\ldots 10=$ Very different

What is your overall opinion of the scar

compared to normal skin? 